\title{
Calendar of Events
}

February 5-8, 2009

Integrating Psychosocial Research and Practice in Quality Cancer Care:

Setting the Standard

Location: Westin Charlotte

Charlotte, North Carolina, USA

Contact: American Psychosocial Oncology Society (APOS)

2365 Hunters Way, Charlottesville, VA 22911, USA

Phone: + 1 434-293-5350

Fax: + 1 434-977-1856

E-mail: info@apos-society.org

Website: www.apos-society.org

http://www.apos-society.org/professionals/meetings-ed/

annualconference.aspx

March 25-28, 2009

American Academy of Hospice and Palliative Medicine The AAHPM/HPNA Annual Assembly

Location: Austin, Texas

Contact: American Academy of Hospice and Palliative Medicine

4700 W. Lake Ave. Glenview, IL 60025

Tel: 847-375-4712

Fax: 847-375-6475

E-mail: info@aahpm.org

May 7-10, 2009

11th Congress of the European Association for Palliative Care

Location: Vienna, Austria

Contact: Heidi Blumhuber

EAPC Onlus

Istituto Nazionale dei Tumori

20133 Milano, Italy
E-mail: heidi.blumhuber@istitutotumori.it

Web: www.eapcnet.org

June 21-25, 2009

International Psycho-Oncology Society (IPOS)

11th World Congress of Psycho-Oncology

Multi-disciplinary Perspectives in Psycho-Oncology:

Interaction and Integration

Location: Vienna, Austria

Website: www.ipos-society.org/ipos2009

\author{
June 25-27, 2009 \\ MASCC/ISOO \\ Supportive Care in Cancer \\ 2009 International Symposium \\ Location: Rome, Italy \\ Contact: Business Services International, Inc. \\ Phone: $760-480-2525$ \\ Fax: 760-480-2544 \\ E-mail: debbiekurth@bsiincorp.com
}

\section{September 23-26, 2009}

The International College of Psychosomatic Medicine ICPM

20th World Congress on Psychosomatic Medicine

Location: Torino, Italy

Contact: Organizing Secretariat

Centro Congressi Internazionale

Corso Bramante, 58/9 - 10126

Turin, Italy

Phone: +39 011-2446911

Fax: + 39 011-2446900

E-mail: info@icpm2009.com

Website: www.icpm2009.com 